\title{
Four Year'Trend of Carbapenem-Resistance in Newly Opened ICUs of a University-Affiliated Hospital of South Korea
}

Bo Min Kim, M.D., Eun Ju Jeon, M.D., Ju Young Jang, M.D., Jin-Won Chung, M.D., Jihoon Park, M.D., Jae Chol Choi, M.D., Jong Wook Shin, M.D., In Won Park, M.D., Byoung Whui Choi, M.D., Jae Yeol Kim, M.D. Division of Pulmonary and Allergy, Department of Internal Medicine, Chung-Ang University College of Medicine, Seoul, Korea

Background: Carbapenem-resistance is rapidly evolving among the pathogenic microbes in intensive care units (ICUs). This study aimed to determine annual trend of carbapenem-resistance in the ICU for 4 years, since the opening of a university-affiliated hospital in South Korea.

Methods: From 2005 to 2008, microbial samples from consecutive 6,772 patients were screened in the ICU. Three hundred and ninety-seven patients (5.9\%) and their first isolates of carbapenem-resistant pathogens were analyzed. Results: The percentage of patients infected with carbapenem-resistant organisms increased constantly during the initial three years $(2.3 \%$ in $2005,6.2 \%$ in $2006,7.8 \%$ in 2007), then it declined to $6.5 \%$ in 2008. Acute Physiology and Chronic Health Evaluation (APACHE) III score at admission was 58.0 \pm 23.5 , the median length of the ICU stay was 37 days, and the mortality rate was 37.5\%. The sampling sites were endotracheal suction (67\%), catheterized urine (17\%), wound (6\%) and others (10\%). Bacteria with carbapenem-resistance were Pseudomonas aeruginosa (247 isolates, 62\%), Acinetobacter baumannii (117 isolates, 30\%), Enterobacteriaceae (12 isolates, 3\%), and others $(21,5 \%)$. Of note, peak isolation of carbapenem-resistant microorganisms in medical ICU was followed by the same epidemic at surgical ICU.

Conclusion: Taken together, carbapenem-resistant pathogens are of growing concern in the ICU.

Key Words: Carbapenems; Drug Resistance, Bacterial; Intensive Care Units

\section{Introduction}

Multidrug-resistant (MDR) strains have become progressively more common causes of nosocomial infections since $1980 s^{1}$. Many Asian countries, including Republic of Korea, have also shown increasing rates of antibiotic resistance ${ }^{2}$. Especially, intensive care units (ICU) have become the main foci for developing multidrug-resistance such as methicillin-resistant Staphylococcus aureus, vancomycin-resistant Enterococci, and mul-

Address for correspondence: Jae Yeol Kim, M.D. Division of Allergy, Respiratory and Critical Care Medicine, Department of Internal Medicine, Chung-Ang University Hospital, Chung-Ang University College of Medicine, 224-1, Heukseok-dong, Dongjak-gu, Seoul 156-756, Korea

Phone: 82-2-6299-1396, Fax: 82-2-825-7571

E-mail: jykimmd@cau.ac.kr

Received: Dec. 1, 2011

Revised: Jan. 26, 2012

Accepted: Mar. 15, 2012 ti-drug resistant gram negative bacilli ${ }^{3}$.

Among these pathogens, gram-negative bacteria resistant to carbapenem are great concern. Carbapenems are the drugs of choice for the treatment of infection by many gram-negative bacteria ${ }^{4}$. However, the increasing use of carbapenems has contributed to the development of carbapenem-resistance among these pathogens. The most frequently encountered microorganisms resistant to carbapenems are Pseudomonas aeruginosa, Acinetobacter baumannii, and Enterobacteriaceae.

$P$. aeruginosa causes severe invasive diseases in critically ill and immunocompromised patients. Pseudomonas species isolated from patients in ICUs show carbapenem-resistance rates of $28 \sim 37 \%$, . A. baumannii is also a major cause of nosocomial infections. Acinetobacter initially exhibited almost uniform susceptibility to imipenem in most institutions. However, carbapenem-resistant strains are emerging worldwide and resist- 
ance rates have reached $50 \%$ to $60 \%$ in some institutions ${ }^{7,8}$. Among the Enterobacteriaceae, extendedspectrum beta-lactamase-producing Escherichia coli and Klebsiella pneumoniae which are resistant to carbapenems are great problems in ICUs because carbapenems are usually considered the last choice for the treatment of these organisms? ${ }^{9}$. The major driving force behind carbapenem-resistance has been the heavy use of third generation cephalosporins, aztreonam, and imipenem.

Although there have been many articles regarding MDR pathogens in the ICU, few have evaluated the annual trend of carbapenem-resistance since the opening of new ICU. This study aimed to determine the annual trend of carbapenem-resistance patterns of major pathogens and their respective contribution at a newly opened ICU of a university-affiliated hospital in Seoul, Republic of Korea.

\section{Materials and Methods}

\section{Objective patients}

We retrospectively reviewed the clinical data of eligible patients who had been admitted and taken clinical samples for microbiologic test at 21-bed medical and 16-bed surgical ICU of Chung-Ang University Hospital between January 2005 and December 2008. This study was approved by Institutional Review Board (IRB) of Chung-Ang University Hospital.

\section{Definition of carbapenem resistance}

Carbapenem-resistance was defined as minimum inhibitory concentration equals to or exceeds $16 \mu \mathrm{g} / \mathrm{mL}$ for imipenem or meropenem, which were examined by antibiotic susceptibility testing with the VITEK-2 system (Biomerieux, Lyon, France).

\section{Clinical characteristics analyzed}

We reviewed the following clinical characteristics: age, gender, underlying diseases, the routes of admission, the duration of ICU stay, the disease severity according to the Acute Physiology and Chronic Health Evaluation (APACHE) III scoring system, the previous use of antibiotics and their adequacy before ICU admission, and mortality rate. With this baseline data, annual trend in the number of patients infected with carbapenem-resistant pathogens were evaluated. The annual trend was sub-analyzed according to respective pathogen with carbapenem-resistance and to the sites of admission (medical intensive care unit [MICU] vs. surgical intensive care unit [SICU]). As for microbiologic characteristics, sites of isolation and drug sensitivity patterns were evaluated.

\section{Annual consumption of representative antimicrobial agents at Chung-Ang University Hospital}

Annual consumption of representative antibiotics used in our hospital has been calculated from 2006 to 2008. The use of antibiotic agents is expressed as defined daily doses per 1,000 patients per day ${ }^{10}$.

\section{Statistical analysis}

For statistical analysis, the SPSS version 10.0 (SPSS Inc., Chicago, IL, USA) program was used. Statistically significant differences were accepted at p-values below 0.05. Student's t-test was applied for comparison between the continuous variables and Pearson's Chisquare test was used for the discrete variables.

\section{Results}

\section{Characteristics of the study patients}

From January 2005 to December 2008, there were 6,772 consecutive patients admitted to MICU or SICU of Chung-Ang University Hospital. Three hundred and ninety-seven patients (5.9\%) and their firstly isolated carbapenem-resistant bacteria were evaluated. The age of patients (mean $\pm \mathrm{SD}$ ) infected with carbapenem-resistant bacteria was $65 \pm 18$ years. Male patients comprised $65.7 \%$ of them. Many of them were admitted via emergency department. Over one hundred seventy patients had diabetes mellitus as an underlying disease. The patients admitted to MICU slightly overnumbered those admitted to SICU. The median duration of the ICU stay was 37 days (range, $2 \sim 1,320$ days). The APACHE 
BM Kim et al: Carbapenem resistance in the ICU: 4-year experience

III score (mean \pm SD) was $57.1 \pm 22.8$ and in-hospital mortality rate was $37.5 \%$. In details, the mortality rate of patients infected with carbapenem-resistant $P$. aeruginosa was $32.0 \%$ and it was $37.9 \%$ with carbapenem-resistant $A$. baumannii. Most of them were previously treated with antibiotics before ICU admission (350 patients, 88.2\%) and only part of the initial antibiotics were adequate to cover pathogens with carbapenem-resistance $(37,9.3 \%)$ (Table 1$)$. The common reasons for culture were leukocytosis (215/397, 54.2\%), fever (156/ $397,39.3 \%)$, and new infiltration in chest X-ray (90/397, $22.7 \%)$.

\section{Characteristics of carbapenem-resistant patho- gens}

Organisms with carbapenem-resistance were $P$. aerzginosa (247 isolates, 62.2\%), A. baumannii (117 isolates, $29.5 \%$ ), Enterobacteriaceae (12 isolates, 3.0\%) and others (21 isolates, 5.3\%) (Figure 1). Two-hundred and ten pathogens showed resistance both to imipenem and to meropenem. Of 247 isolates of $P$. aeruginosa, 15 isolates were resistant to all antibiotics tested. One-hundred ninety-nine isolates $(80.6 \%)$ were sensitive to colistin. As for $A$. baumannii, 2 were resistant to all antibiotics, while 85 isolates $(72.6 \%)$ were sensitive to

Table 1. Demographics and clinical characteristics of patients with carbapenem resistance

\begin{tabular}{lc}
\hline \multicolumn{1}{c}{ Characteristics } & Results \\
\hline No. & 397 \\
Age, yr & $65.5 \pm 18.0$ \\
Male & $261(65.7)$ \\
Diabetes mellitus & $171(43.1)$ \\
MICU:SICU & $227(57.2): 170(42.8)$ \\
Route of admission (ER:Ward) & $281(70.8): 116(29.2)$ \\
Length of ICU stay, days & $37(2 \sim 1,320)$ \\
Use of previous antibiotics & $350(88.2)$ \\
Appropriate antibiotics & $37(9.3)$ \\
APACHE III score & $57.1 \pm 22.9$ \\
Mortality rate & $36.5 \%$ \\
\hline
\end{tabular}

Data are presented as numbers (\%) unless otherwise indicated. MICU: medical intensive care unit; SICU: surgical intensive care unit; ER: emergency room; APACHE: Acute Physiology and Chronic Health Evaluation. colistin. Transtracheal aspirates (67\%) were the most common sources of carbapenem-resistant organisms followed by catheterized urine (17\%), wound (6\%), sputum $(3 \%)$, blood $(2 \%)$, central venous catheter $(1 \%)$, and others (4\%) (Figure 2).

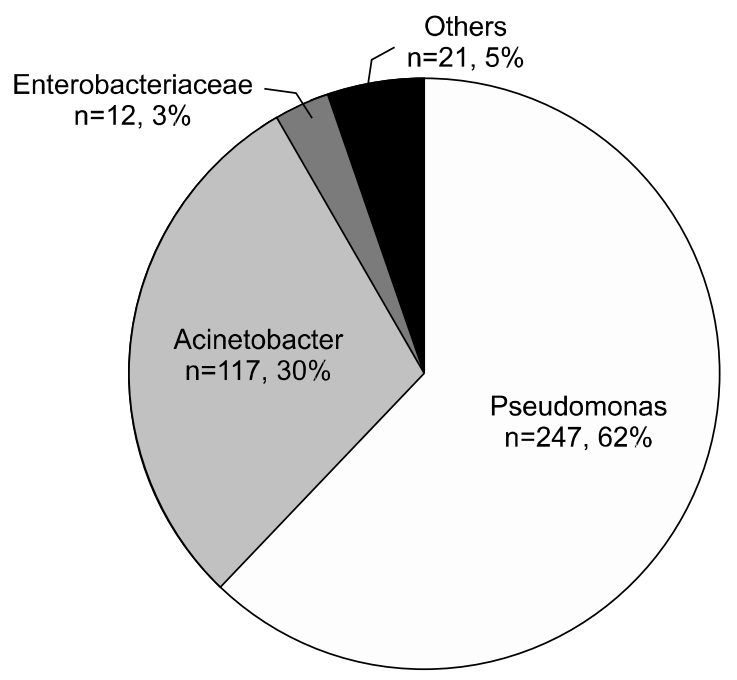

Figure 1. The proportions of bacterial etiologies with carbapenem-resistnace isolated in intensive care unit from 2005 to 2008. Pseudomonas: Pseudomonas aeruginosa; Acinetobacter: Acinetobacter baumannii, Enterobacteriaceae: Enterobacter, Klebsiella, Proteus, and Serratia.

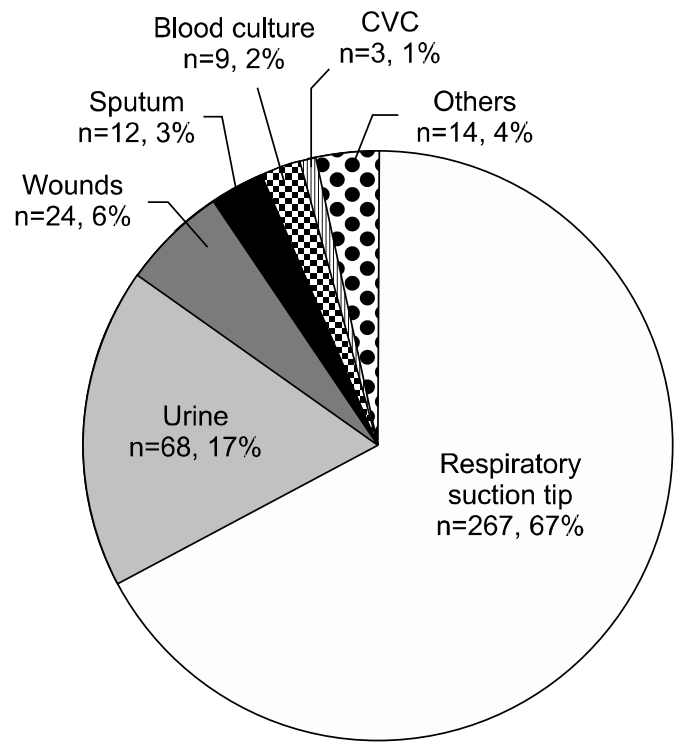

Figure 2. The sites of the isolated carbapenem-resistant pathogens in the patients admitted to the intensive care unit. CVC: central venous catheter. 


\section{Chronological profile of infection with carbapenem- resistant pathogens}

Carbapenem-resistant $P$. aeruginosa was first detected at the 38th day since the opening of the ICU. The percentage of patients infected with carbapenem-resistant organisms increased constantly during the initial three years, then the resistance rate declined slightly in 2008 (Figure 3). Of note, A. baumannii infection was not significant in the first two years. However, after explosive isolation on 3rd quartile of 2007, there was sustained isolation of $A$. baumannii until the 4th quartile of 2008

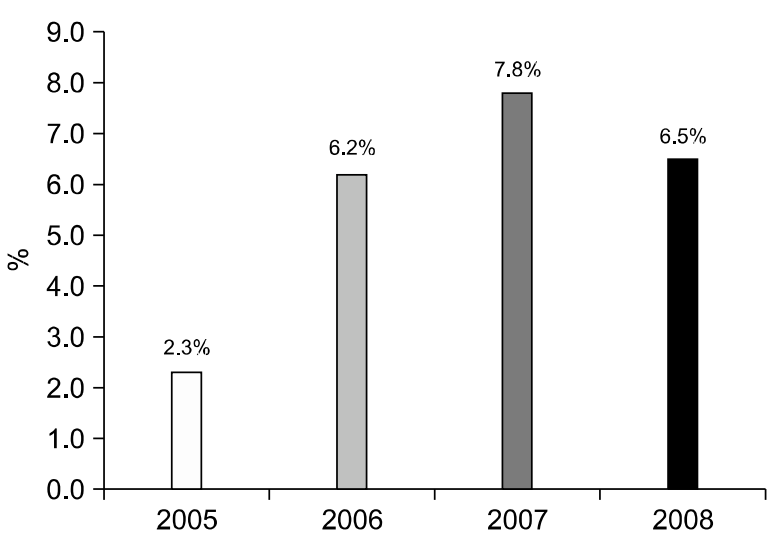

Figure 3. Annual trend of percentage of patients infected with carbapenem-resistant pathogens in the intensive care unit from 2005 to 2008.

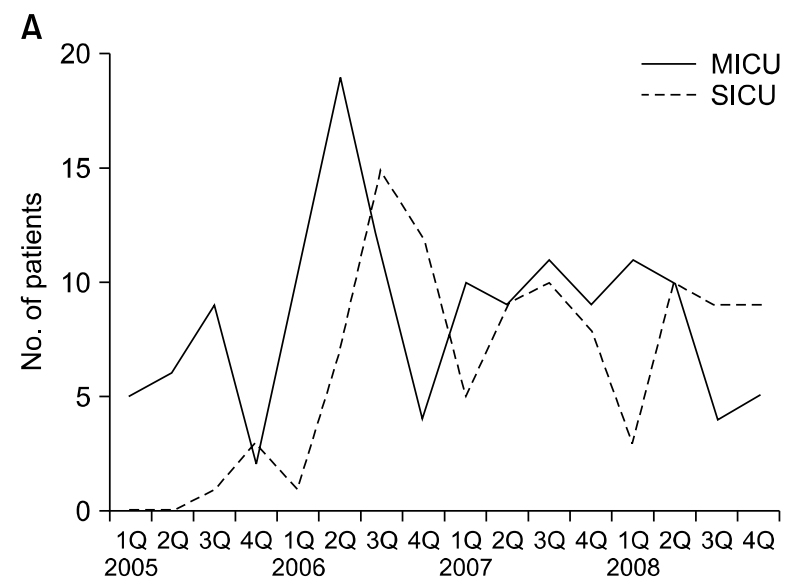

(Figure 4). Interestingly, the peak of infection of $P$. aeruginosa and $A$. baumannii in the MICU was followed by same epidemics in the SICU (Figure 5).

\section{Annual consumption of representative antimicrobial agents at Chung-Ang University Hospital from 2006 to 2008}

Annual consumption of many antibiotics including cefazolin, cetotaxime, cefepime, ciprofloxacin, moxifloxacin, imipenem, meropenem, vancomycin, peperacillin/

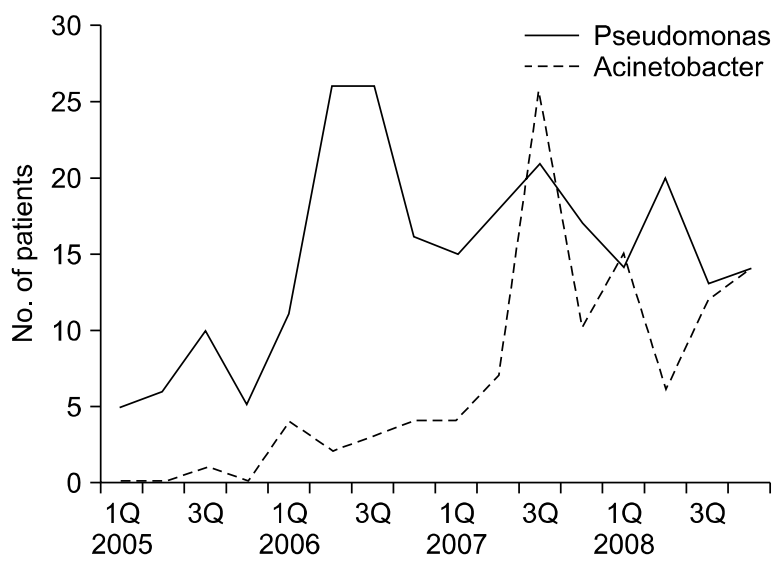

Figure 4. The number of patients infected with Pseudomonas aeruginosa, Acinetobacter baumannii, or Enterococcus faecium in the intensive care unit from 2005 to 2008 with 3-month interval. Pseudomonas: P. aeruginosa; Acinetobacter: A. baumannii.

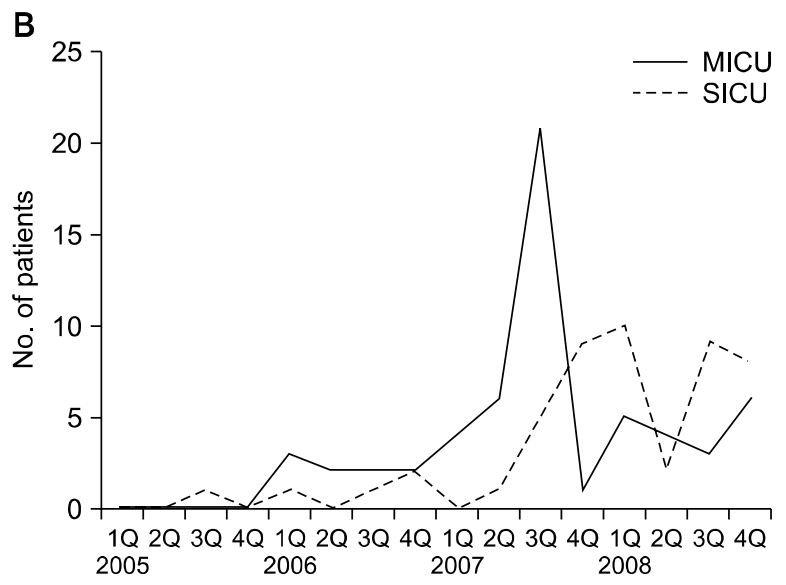

Figure 5. The number of patients infected either with Pseudomonas aeruginosa (A) or Acinetobacter baumannii (B) according to the site of admission from 2005 to 2008 with 3-month interval. MICU: medical intensive care unit; SICU: surgical intensive care unit. 
BM Kim et al: Carbapenem resistance in the ICU: 4-year experience

Table 2. Annual consumption of representative antimicrobial agents (DDD/1,000 patient-day) at Chung-Ang University Hospital from 2006 to 2008

\begin{tabular}{lrrr}
\hline Antibiotic agents & 2006 & 2007 & 2008 \\
\hline Amikacin & 4.07 & 4.38 & 4.87 \\
Cefotaxim & 9.39 & 14.32 & 18.17 \\
Cefepime & 0.57 & 4.45 & 7.81 \\
Ciprofloxacin & 22.70 & 26.63 & 33.38 \\
Clindamycin & 7.42 & 8.25 & 7.37 \\
Ceftriaxone & 90.62 & 126.33 & 87.00 \\
Cefazolin & 2.10 & 4.39 & 4.40 \\
Gentamycin & 3.83 & 4.72 & 4.47 \\
Isepamicin & 39.53 & 46.51 & 32.18 \\
Levofloxacin & 14.38 & 23.00 & 20.43 \\
Moxifloxacin & 0.73 & 11.71 & 20.24 \\
Metronidazole & 40.25 & 66.40 & 58.43 \\
Imipenem & 4.44 & 6.48 & 8.65 \\
Meropenem & 7.03 & 11.63 & 15.43 \\
Ceftazidime & 3.69 & 5.28 & 4.30 \\
Teicoplanin & & 2.95 & 3.87 \\
Vancomycin & 6.62 & 12.04 & 16.86 \\
Piperacillin/Tazobactam & 0.06 & 12.13 & 12.46 \\
Ampicillin/Sulbactam & 17.20 & 20.04 & 15.68 \\
\hline
\end{tabular}

DDD: defined daily doses.

tazobactam, and amikacin has increased constantly from 2006 to 2008 (Table 2).

\section{Discussion}

Carbapenems are one of beta-lactam drugs which contain bicyclic beta-lactam ring in the center. Although they share a common beta-lactam ring with penicillin, they are generally resistant to cleavage by most plasmid and chromosomal beta-lactamase ${ }^{11}$. Therefore, they retain activity against the chromosomal cephalosporinases and extended-spectrum beta-lactamases found in many gram-negative pathogens. However, the increasing use of carbapenems has contributed to the development of carbapenem-resistance in many pathogens ${ }^{12}$. In the present study, we evaluated annual trend of carbapenem-resistance at newly opened ICUs of a universityaffiliated hospital for 4 years since 2005. We found that carbapenem-resistance increased constantly during the first 3 years and then decreased slightly in the fourth year in the ICU (Figure 3). As expected, annual con- sumption of imipenem and meropenem has increased constantly along with other important antibiotics (betalactam/beta-lactase inhibitors, cephalosporins, fluoroquinolones, and aminoglycosides) in our hospital from 2006 to 2008 (Table 2). Therefore, it is not surprising that even though standardized infection control measures have been applied at our ICUs, they could not prevent the proliferation of carbapenem-resistant pathogens. It is a great concern because optimal treatment of infection due to carbapenem-resistant organisms is uncertain and antibiotics options are very limited. It is well reflected in our data that only part of the initial antibiotics were adequate to cover pathogens with carbapenem-resistance $(n=37,9.3 \%)$ (Table 1$)$.

Risk factors for developing multi-drug resistance are old age, male gender, cardiovascular diseases, mechanical ventilation, duration of the ICU stay, surgery, trau$\mathrm{ma}$, and the use of broad-spectrum antibiotics ${ }^{13}$. It is consistent with our finding that most of patients with carbapenem-resistance (88.2\%) have been treated by other antibiotics before ICU admission, had long duration of ICU stay, high APACHE III score, and high mortality rate of $37.5 \%$ (Table 1 ). It is noteworthy that over forty percent of patients had diabetes as an underlying disease.

$P$. aeruginosa and $A$. baumannii were most common pathogens with carbapenem-resistance, as expected (Figure 1). It is consistent with the fact that Pseudomonas is the most widespread MDR gram-negative pathogen causing pneumonia in hospitalized patients ${ }^{14}$. $P$. aeruginosa and Acinetobacter have the potential of causing outbreaks in the hospital setting and most outbreaks occurred in adult ICUs ${ }^{15,16}$. Unfortunately it is evident that our ICUs are not the exception. It is somehow relieving that many isolates of $P$. aeruginosa (80.6\%) and $A$. baumannii (72.6\%) remain sensitive to colistin. However considering the high degree of nephrotoxicity, colistin as an alternative for the treatment of carbapenem-resistant pathogens is not a great relief.

Pseudomonas is the most common pathogen of hospital-acquired respiratory tract infection and the most common manifestation of Acinetobacter outbreak in- 
fection have been nosocomial pneumonia (primarily ventilator-associated) ${ }^{17}$. It is consistent with our findings that respiratory specimen were the most common source of isolation for carbapenem-resistant pathogens (Figure 2).

Carbapenem-resistant rate increased constantly during the first 3 years since the opening of ICUs (Figure 3). Our ICUs adopted standardized, meticulous infection control measures including, use of gown, glove, facial masks for the care of patients, repeated education for prevention of infection in the ICU, monitoring of hand washing among medical staffs, cohort isolation of patients infected by MDR pathogens, closed suction system of endotracheal tube, and oral hygiene using chlorhexidine three times a day. With all these measures, proliferation of carbapenem-resistant pathogens in the ICU seems to be an unavoidable phenomenon.

A. baumannii infection was not significant in the first two years. However, after explosive isolation on $3 \mathrm{rd}$ quartile of 2007, there was sustained isolation of $A$. baumannii until the 4th quartile of 2008 (Figure 4). Once the epidemic of $A$. baumanni happens, it is very difficult to eliminate it from the ICU. Koeleman et al. ${ }^{18}$ even suggested the complete 10-day closure of ICU to contain an $A$. baumannii. Although we did not adopt this policy during the period, it could be an option when there is no other way to control explosive isolation of $A$, baumannii in the ICU.

Of note, peak isolation of $P$. aeruginosa or $A$. baumannii in the MICU was followed by same epidemics at SICU (Figure 5). In our hospital, MICU with 21 beds and SICU with 16 beds are run side-by- side on the same floor. Although each ICU has its own operating staffs, sometimes doctors can take care of both sides of patients. Still, the direction of spread was always from MICU to SICU but not vice versa. It can be explained that generally patients in the MICU have more risk factors for MDR pathogens than those in the SICU.

This study has several limitations. First, we did not evaluate the risk factors for carbapenem-resistant organism systematically. Second, the isolated pathogen could be colonization. For example, $A$, baumannii is a ubiq- uitous commensal and it is widely distributed in ICU environments. Differentiation between colonization and true infection may be difficult. However, clinical samples were collected when the patients developed signs of infection. Therefore, the possibility of colonization might be low. In spite of some limitations, the present study is a rare report that presented the annual trend of carbapenem-resistance in newly-opened ICUs for a long period of time. In conclusion, carbapenem-resistant pathogens are growing concern in the ICUs and epidemic of carbapenem-resistance in the MICU can be followed subsequent epidemic in the SICU by the same pathogens when they are run in close proximity.

\section{References}

1. Gaynes R, Edwards JR; National Nosocomial Infections Surveillance System. Overview of nosocomial infections caused by gram-negative bacilli. Clin Infect Dis 2005; $41: 848-54$

2. Song JH. Current status and future strategies of antimicrobial resistance in Korea. Korean J Med 2009;77: 143-51.

3. Neuhauser MM, Weinstein RA, Rydman R, Danziger LH, Karam G, Quinn JP. Antibiotic resistance among gram-negative bacilli in US intensive care units: implications for fluoroquinolone use. JAMA 2003;289: 885-8.

4. Paterson DL, Ko WC, Von Gottberg A, Mohapatra S, Casellas JM, Goossens H, et al. Antibiotic therapy for Klebsiella pneumoniae bacteremia: implications of production of extended-spectrum beta-lactamases. Clin Infect Dis 2004;39:31-7.

5. Lee K, Lee HS, Jang SJ, Park AJ, Lee MH, Song WK, et al. Antimicrobial resistance surveillance of bacteria in 1999 in Korea with a special reference to resistance of enterococci to vancomycin and gram-negative bacilli to third generation cephalosporin, imipenem, and fluoroquinolone. J Korean Med Sci 2001;16:262-70.

6. Dubois V, Arpin C, Melon M, Melon B, Andre C, Frigo $\mathrm{C}$, et al. Nosocomial outbreak due to a multiresistant strain of Pseudomonas aeruginosa P12: efficacy of cefepime-amikacin therapy and analysis of beta-lactam resistance. J Clin Microbiol 2001;39:2072-8.

7. Rhomberg PR, Jones RN. Contemporary activity of meropenem and comparator broad-spectrum agents: MYSTIC program report from the United States compo- 
BM Kim et al: Carbapenem resistance in the ICU: 4-year experience

nent (2005). Diagn Microbiol Infect Dis 2007;57:207-15.

8. Agodi A, Zarrilli R, Barchitta M, Anzaldi A, Di Popolo A, Mattaliano A, et al. Alert surveillance of intensive care unit-acquired Acinetobacter infections in a Sicilian hospital. Clin Microbiol Infect 2006;12:241-7.

9. Winokur PL, Canton R, Casellas JM, Legakis N. Variations in the prevalence of strains expressing an extended-spectrum beta-lactamase phenotype and characterization of isolates from Europe, the Americas, and the Western Pacific region. Clin Infect Dis 2001;32 Suppl 2:S94-103.

10. World Health Organization. WHO Collaborating Centre for Drug Statistics Methodology [Internet]. Oslo: WHO; 2011 [cited 2011 Dec 1]. Available from: http://www. whocc.no/atcddd/.

11. Norrby SR. Carbapenems. Med Clin North Am 1995; 79:745-59.

12. Brown S, Amyes S. OXA (beta)-lactamases in Acinetobacter: the story so far. J Antimicrob Chemother 2006;57:1-3.

13. Lortholary O, Fagon JY, Hoi AB, Slama MA, Pierre J, Giral P, et al. Nosocomial acquisition of multiresistant Acinetobacter baumannii: risk factors and prognosis. Clin Infect Dis 1995;20:790-6.
14. Richards MJ, Edwards JR, Culver DH, Gaynes RP. Nosocomial infections in medical intensive care units in the United States. National Nosocomial Infections Surveillance System. Crit Care Med 1999;27:887-92.

15. Crespo MP, Woodford N, Sinclair A, Kaufmann ME, Turton J, Glover J, et al. Outbreak of carbapenem-resistant Pseudomonas aeruginosa producing VIM-8, a novel metallo-beta-lactamase, in a tertiary care center in Cali, Colombia. J Clin Microbiol 2004;42:5094-101.

16. Agarwal R, Gupta D, Ray P, Aggarwal AN, Jindal SK. Epidemiology, risk factors and outcome of nosocomial infections in a Respiratory Intensive Care Unit in North India. J Infect 2006;53:98-105.

17. Pournaras S, Markogiannakis A, Ikonomidis A, Kondyli L, Bethimouti K, Maniatis AN, et al. Outbreak of multiple clones of imipenem-resistant Acinetobacter baumannii isolates expressing OXA-58 carbapenemase in an intensive care unit. J Antimicrob Chemother 2006; 57:557-61.

18. Koeleman JG, Parlevliet GA, Dijkshoorn L, Savelkoul PH, Vandenbroucke-Grauls CM. Nosocomial outbreak of multi-resistant Acinetobacter baumannii on a surgical ward: epidemiology and risk factors for acquisition. J Hosp Infect 1997;37:113-23. 